\title{
The Human Genetic Variants CYP2J2 rs2280275 and EPHX2 rs75II4I and Risk of Diabetic Nephropathy in Egyptian Type 2 Diabetic Patients
}

This article was published in the following Dove Press journal: The Application of Clinical Genetics

\author{
Mona S Habieb $\mathbb{D}^{\prime}$ \\ Ashraf A Dawood' \\ Mahmoud M Emara ${ }^{2}$ \\ Mohammad G Elhelbawy ${ }^{3}$ \\ Nesreen G Elhelbawy' \\ 'Medical Biochemistry \& Molecular \\ Biology Department, Faculty of Medicine, \\ Menoufia University, Shebin Elkom City, \\ Egypt; ${ }^{2}$ Internal Medicine Department, \\ Faculty of Medicine, Menoufia University, \\ Shebin Elkom City, Egypt; ${ }^{3}$ Clinical \\ Pathology Department, Faculty of \\ Medicine, Menoufia University, Shebin \\ Elkom City, Egypt
}

Background: Diabetic nephropathy (DN), the primary driver of end-stage kidney disease, is a problem with serious consequences for society's health. Single nucleotide polymorphisms (SNPs) can define differences in susceptibility to DN and aid in development of personalized treatment. Giving the importance of epoxyeicosatrienoic acids (EETs) in kidney health, we aimed to study the association between two SNPs in the genes controlling synthesis and degradation of EETs (CYP2J2 rs2280275 and EPHX2 rs751141 respectively) and susceptibility of type 2 diabetes mellitus (T2DM) patients to develop DN.

Patients and Methods: Two hundred subjects were enrolled and categorized into three groups: group I (80 T2DM patients with DN), group II (60 T2DM patients without DN) and group III (60 healthy controls). Urea, creatinine, albumin/creatinine ratio (ACR), and eGFR were measured for all participants. Genotyping of CYP2J2 rs2280275 and EPHX2 rs751141 was done by real time PCR.

Results: There was no significant difference between the studied groups regarding CYP $2 J 2$ rs2280275. In contrast, EPHX2 rs751141 was associated with increased risk of DN under a dominant model (GG vs GA+AA: OR=0.375; 95\% CI (0.19-0.75), $\mathrm{P}=0.006$ ) in unadjusted model and after adjustment for age and sex $(\mathrm{OR}=0.440 ; 95 \% \mathrm{CI}(0.21-0.92), \mathrm{P}=0.029)$, recessive model (AA vs $\mathrm{GG}+\mathrm{GA}$ : $\mathrm{OR}=0.195 ; 95 \% \mathrm{CI}(0.05-0.74), \mathrm{P}=0.017$ ) and additive model (GA vs GG+AA): OR=0.195; 95\% CI (0.05-0.74), $\mathrm{P}=0.017)$.

Conclusion: CYP2J2 rs2280275 was not associated with DN predisposition. However, $E P H X 2$ rs751141 could be a genetic marker for development and progression of DN among Egyptian T2DM patients.

Keywords: diabetic nephropathy, $C Y P 2 J 2, E P H X 2$, real time PCR

\section{Introduction}

Diabetic nephropathy (DN), one of the microvascular complications of diabetes mellitus (DM), is the primary single driver of end-stage kidney disease. ${ }^{1}$ It is characterized by accumulation of advanced glycation end products and growth factors, aside from hemodynamic and hormonal disturbances, which contribute to proteinuria, hypertension, and progressive deterioration of glomerular filtration. ${ }^{2}$ Not all diabetics develop DN, suggesting that individual genetic differences may be implicated in incidence and progression of DN. However, the total genetic profile remains elusive. ${ }^{3}$

Epoxyeicosatrienoic acids (EETs) have multiple biological activities in the cardiovascular and renal systems. ${ }^{4}$ Renal EETs have anti-inflammatory,
Correspondence: Nesreen G Elhelbawy Medical Biochemistry and Molecular Biology Department, Faculty of Medicine, Menoufia University, Shebin Elkom City 325I I, Egypt

Tel +201069590533

Fax +20482181009

Email nesrin.elhelbawy@yahoo.com
The Application of Clinical Genetics 2020:13 165-178

DovePress f in $\mathbf{P}$ 
anti-apoptotic, proangiogenic, fibrinolytic and anti-hypertensive roles. ${ }^{5}$ Therefore, EETs are often termed renoprotective agents. ${ }^{6}$ EETs are formed from arachidonic acid by the action of cytochrome P450 (CYP) epoxygenase enzymes. The main EETs producers are CYP2C and CYP2J subfamilies ${ }^{7}$ which show higher expression in the proximal tubules of the kidney. ${ }^{8} \mathrm{CYP} 2 \mathrm{~J}$ subfamily contains only one member termed CYP2J2. ${ }^{9}$ Experimentally, over expression of CYP2J2 has been considered a safeguarding factor against kidney damage. ${ }^{10}$ Genetic variations of CYP2J2 gene affect its expression throughout the population. ${ }^{11}$ One of them is the CYP2J2 rs 2280275 single nucleotide polymorphism (SNP) situated in the intronic region of CYP2J2 gene. This SNP is in linkage disequilibrium with the promoter SNP rs890293 which results in reduced levels of CYP2J2 epoxygenase metabolites in vivo. $^{11,12}$

Previous studies had documented the association of CYP2J2 rs2280275 with cardiovascular diseases such as coronary heart diseases ${ }^{13}$ and hypertension. ${ }^{14}$

Up to the present time, no studies have been conducted to analyze the implication of CYP2J2 rs2280275 in susceptibility to DN.

The bioavailability of EETs depends not only on the rate of synthesis but also on the fact that they are quickly hydrolyzed to less active metabolites by soluble epoxide hydrolase (sEH) enzyme. ${ }^{15} \mathrm{sEH}$ is a cytosolic enzyme that is expressed in many tissues, including kidney and liver. ${ }^{7}$ In many experimental studies, it was found that the inhibition of $\mathrm{SEH}$ had a renoprotective effect. ${ }^{16,17}$ sEH is encoded by EPHX2 gene. ${ }^{18}$ A common SNP in the EPHX2 gene is the functional rs751141 G/A polymorphism. The variant Aallele of that SNP results in an sEH enzyme with $25-58 \%$ inadequate catalytic activity. ${ }^{19}$

The role of EPHX2 polymorphisms in cardiovascular diseases has gained attention in many studies. ${ }^{20}$ However, a limited number of studies have examined this SNP in kidney disorders, ${ }^{6,21,22}$ with only one study performed in diabetic kidney disease. ${ }^{23}$

Considering the value of EETs in the kidney, we speculated that sequence variations within CYP2J2 and EPHX2 might have an impact on DN predisposition. In view of this, we designed this study to investigate the association of CYP2J2 rs2280275 and EPHX2 rs751141 with DN in Egyptian T2DM patients in relation to kidney function parameters.

\section{Materials and Methods}

\section{Participants and Data Collection}

This study was completed by collaboration between Medical Biochemistry and Molecular Biology, Internal Medicine and Clinical Pathology Departments; Faculty of Medicine, Menoufia University, Egypt in the period from July 2018 to June 2019. This study included 140 T2DM patients (80 patients with DN [group I] and 60 patients without DN [group II]) and 60 apparently healthy control subjects [group III]. Patients were selected from the nephrology unit at the Internal Medicine Department, Menoufia University Hospital with history of DN present for more than 3 months (group I) with a decreased eGFR $<60 \mathrm{~mL} / \mathrm{min} / 1.73 \mathrm{~m}^{2}$ and albumin/creatinine ratio $(\mathrm{ACR})>30 \mathrm{mg} / \mathrm{g}$. Diagnosis of T2DM was based on the recommendations of the American Diabetes Association; fasting blood glucose $\geq 126 \mathrm{mg} / \mathrm{dL}$ or $2 \mathrm{~h}$ blood glucose after overload with $75 \mathrm{~g}$ of glucose $\geq 200$ $\mathrm{mg} / \mathrm{dL}$ in oral glucose tolerance test or glycated hemoglobin (HbA1c) $\geq 6.5 \%$ in patients with classic symptoms of hyperglycemia (American Diabetes Association, 2015) ${ }^{24}$ Group II included 60 T2DM patients without DN with ACR $<30 \mathrm{mg} /$ g. Hypertensive patients were known to have long-standing hypertension (systolic blood pressure $\geq 140 \mathrm{mmHg}$ and/or diastolic blood pressure $\geq 90 \mathrm{mmHg}$ in at least two separate measurements or regular use of antihypertensive medication). ${ }^{25}$ Exclusion criteria for both groups included other primary or secondary renal diseases, malignancy, hepatic or cardiac failure and severe infections.

This study was conducted in accordance with the Declaration of Helsinki.

An informed written consent was obtained from each participant approved by the Ethical Committee of Medical Research, Faculty of Medicine, Menoufia University.

Thorough medical and demographic histories, family history of DM, duration of DM, BMI [calculated as weight $(\mathrm{kg}) /$ height $\left(\mathrm{m}^{2}\right)$ ], smoking, hypertension, systolic blood pressure (SBP), diastolic blood pressure (DBP), fasting blood glucose (FBG), $2 \mathrm{~h}$ postprandial glucose (2h PPG), glycated hemoglobin (HbAlc), total cholesterol (TC), high-density lipoprotein cholesterol (HDL-C), low-density lipoprotein cholesterol (LDL-C), triglycerides (TGs), serum urea, serum creatinine, albumin/creatinine (ACR) and eGFR were obtained for all participants.

\section{Sampling and Biochemical Measurements}

Two types of samples were taken from each participant; blood and urine samples. 


\section{Blood Sampling}

Ten milliliters of venous blood were withdrawn after overnight fasting. Two milliliters of blood were placed in a sodium fluoride containing tube for measurement of blood glucose profile (FBG and $2 \mathrm{~h}$ PPG) by glucose oxidase method (Spinreact diagnostics kit, Girona, Spain) ${ }^{26}$ and $\mathrm{HbA1c}$ by quantitative colorimetric measurement kits as a percentage of total hemoglobin supplied by Techo Diagnostics, Lakeview Ave, Anaheim, CA, USA. ${ }^{27}$ Four milliliters of blood were placed in a plain tube, allowed to clot at room temperature, and centrifuged for $15 \mathrm{~min}$ at $4000 \mathrm{rpm}$. The separated sera were used for measurement of lipid panel (TC, HDL-C, TGs) using the standard enzymatic colorimetric kits (Spinreact diagnostics kit, Spain) ${ }^{28}$ and renal function tests (serum urea and creatinine) using the standard enzymatic colorimetric kits (DIAMOND diagnostics kits, Germany). Friedewal formula was used to calculate LDL-C. ${ }^{29}$ The eGFR was calculated by the Modification of Diet in Renal Disease (MDRD) formula from serum creatinine. ${ }^{30}$ Four milliliters of blood were placed in EDTA tube for DNA extraction and genotyping of $C Y P 2 J 2$ rs 2280275 and $E P H X 2$ rs 751141.

\section{Urine Sampling}

Fifteen milliliters of fresh morning urine samples were collected from every subject in sterile containers for the measurement of creatinine and albumin for calculation of ACR. Urinary albumin was estimated by a solid-phase enzymelinked immunosorbent assay kit (DRG International Inc., USA; cat \# EIA-2361).

\section{Molecular Analysis and Genotyping Assay}

DNA extraction from whole blood was performed by GeneJETGenomic DNA Purification Kit (Thermo Scientific, Lithuania) and quantified by a spectrophotometer.

\section{Genotyping of CYP2J2 Rs2280275 and EPHX2 Rs75II4I}

Genotyping of CYP2J2 Rs2280275 and EPHX2 Rs751141 was done by real-time PCR with allele discrimination using TaqMan ${ }^{\mathbb{B}}$ SNP Genotyping assay kit on Applied Biosystems 7500 real-time PCR with software version 2.0.1 (Applied Biosystems, Foster City, CA). During TaqMan SNP Genotyping, DNA polymerase from the TaqMan Master Mix amplifies target DNA using sequence specific primers. The fluorescence signals for allelic discrimination were provided by two TaqManfluorogenic probes labeled with VIC/ FAM fluorescent dyes. The probe sequences were;
CCTGCTCTGGCCCAGGCAGGTTACC[A/G]GGTCCTA GCTATGGACATGAAAGGC for EPHX2 rs751141, and TCTCTTTCCTCCAATGTCTAGTACA[C/T]GGCACCCC ACTCCCCATGGTAGGTT for CYP2J2 rs2280275 (Applied Biosystems, USA). The total volume of PCR amplification (20 $\mu \mathrm{L})$ consisted of: $10 \mu \mathrm{L}$ of $2 \mathrm{x}$ TaqMan Genotyping Master Mix (Thermo Scientific), $1.25 \mu \mathrm{L}$ of 20x SNP assay, $3.75 \mu \mathrm{L}$ of nuclease free water, and $5 \mu \mathrm{L}$ of template DNA. DNase-free water (negative control) was included in each assay run. The PCR cycling conditions were initial denaturation for $10 \mathrm{~min}$ at $95^{\circ} \mathrm{C}$, followed by 50 cycles with denaturation of $15 \mathrm{sec}$ at $94^{\circ}$ $\mathrm{C}$ and $60 \mathrm{sec}$ at $60^{\circ} \mathrm{C}$ for annealing and extension. The Applied Biosystems 7500 real-time PCR analyzer measures the fluorescence signal $(\mathrm{Rn})$ from each well of the reading plate. The plotted fluorescence signals indicate which alleles are in each sample and then convert allele calls to genotypes.

\section{Statistics}

The data were analyzed by SPSS version 20 (SPSS Inc. Released 2011. IBM SPSS statistics for windows, version 20.0, IBM Corp, Armonk, NY). The Kolmogorov-Smirnov test was used to verify the normality of distribution of variables. Quantitative data were described using mean, standard deviation (SD), and median. Comparisons between groups for categorical variables were assessed using Chi-squared test (Fisher or Monte Carlo). Student's $t$-test was used to compare two groups for normally distributed quantitative variables while ANOVA was used for comparing the four studied groups and followed by Post Hoc test (Tukey) for pairwise comparison. Kruskal Wallis test was used to compare different groups for abnormally distributed quantitative variables and followed by Post Hoc test (Dunn's for multiple comparisons test) for pairwise comparison. Mann Whitney test was used to compare between two groups for not normally distributed quantitative variables. Odds ratio (OR) was done using Logistic regression analysis and used to calculate the ratio of the odds and $95 \%$ Confidence Interval of an event occurring in one risk group to the odds of it occurring in the non-risk group. Significance of the obtained results was judged at the $5 \%$ level.

\section{Results}

\section{Demographic, Clinical and Biochemical Composition of the Participants}

These results were demonstrated in Table 1 . The three studied groups were matched regarding age $(\mathrm{p}=0.598)$, with mean age of $58.3 \pm 8.5$ years in DN patients. The 
Table I Demographic, Clinical, and Biochemical Parameters

\begin{tabular}{|c|c|c|c|c|c|}
\hline & Group I $(n=80)$ & Group II $(n=60)$ & Group III $(n=60)$ & Test of Sig. & $\mathbf{p}$ \\
\hline \multicolumn{6}{|l|}{ Sex $n(\%)$} \\
\hline Male & $62(77.5 \%)$ & $28(46.7 \%)$ & 29 (48.3\%) & $\chi^{2}=17.962 *$ & $<0.001 *$ \\
\hline Female & $18(22.5 \%)$ & $32(53.3 \%)$ & $31(51.7 \%)$ & & \\
\hline \multicolumn{6}{|l|}{ Age (years) } \\
\hline Mean \pm SD & $58.3 \pm 8.5$ & $57.1 \pm 9.3$ & $58.4 \pm 7.4$ & $\mathrm{~F}=0.5 \mathrm{I} 5$ & 0.598 \\
\hline Family history of DM $n$ (\%) & $70(87.5 \%)$ & 45 (75\%) & 7 (II.7\%) & $\chi^{2}=89.940^{*}$ & $<0.001 *$ \\
\hline \multicolumn{6}{|l|}{ Duration of DM (years) } \\
\hline Mean \pm SD & $13.4 \pm 3.9$ & $15 \pm 5.7$ & - & $\mathrm{t}=1.806$ & 0.074 \\
\hline \multicolumn{6}{|l|}{ BMI $\left(\mathrm{kg} / \mathrm{m}^{2}\right)$} \\
\hline Mean \pm SD & $25.3 \pm 2.1$ & $25.7 \pm 1.7$ & $23.3 \pm 1.9$ & $\mathrm{~F}=28.78 \mathrm{I} *$ & $<0.001 *$ \\
\hline Sig. bet. grps. & \multicolumn{3}{|c|}{$\mathrm{P}_{1}=0.328, \mathrm{P}_{2}<0.00 \mathrm{I}^{*}, \mathrm{P}_{3}<0.00 \mathrm{I}^{*}$} & & \\
\hline Current smokers (\%) & 34 (42.5\%) & $30(50 \%)$ & $15(25 \%)$ & $\chi^{2}=8.348^{*}$ & $0.015^{*}$ \\
\hline History of hypertension (\%) & 45 (56.3\%) & $17(28.3 \%)$ & - & $\chi^{2}=10.830^{*}$ & $0.00 I^{*}$ \\
\hline \multicolumn{6}{|l|}{ SBP (mmHg) } \\
\hline Mean \pm SD & $127.9 \pm 8.5$ & $122.2 \pm 10.9$ & $118.1 \pm 7$ & $\mathrm{~F}=21.445^{*}$ & $<0.001 *$ \\
\hline Sig. bet. grps. & \multicolumn{3}{|c|}{$\mathrm{P}_{1}=0.00 \mathrm{I}^{*}, \mathrm{P}_{2}<0.00 \mathrm{I}^{*}, \mathrm{p}_{3}=0.030 *$} & & \\
\hline \multicolumn{6}{|l|}{ DBP (mmHg) } \\
\hline Mean \pm SD & $79.3 \pm 6.5$ & $76.7 \pm 7.7$ & $76.3 \pm 5$ & $\mathrm{~F}=4.709 *$ & $0.010 *$ \\
\hline Sig. bet. grps. & \multicolumn{3}{|c|}{$\mathrm{p}_{1}=0.046^{*}, \mathrm{p}_{2}=0.017^{*}, \mathrm{p}_{3}=0.939$} & & \\
\hline \multicolumn{6}{|l|}{ FBG (mg/dl) } \\
\hline Mean \pm SD & $196.1 \pm 69.3$ & $203.5 \pm 62.5$ & $80.8 \pm 8.7$ & $\mathrm{~F}=94.853^{*}$ & $<0.001 *$ \\
\hline Sig. bet. grps. & \multicolumn{3}{|c|}{$\mathrm{P}_{1}=0.720, \mathrm{P}_{2}<0.00 \mathrm{I}^{*}, \mathrm{p}_{3}<0.00 \mathrm{I}^{*}$} & & \\
\hline \multicolumn{6}{|l|}{2 h PPG (mg/dl) } \\
\hline Mean \pm SD & $311.9 \pm 77.2$ & $286.7 \pm 54$ & $90.8 \pm 9.3$ & $\mathrm{~F}=285.846^{*}$ & $<0.001 *$ \\
\hline Sig. bet. grps. & \multicolumn{3}{|c|}{$\mathrm{p}_{1}=0.028^{*}, \mathrm{p}_{2}<0.001 *, \mathrm{p}_{3}<0.001 *$} & & \\
\hline \multicolumn{6}{|l|}{ HbAlc (\%) } \\
\hline Mean \pm SD & $8.5 \pm 1.9$ & $8.8 \pm 2.3$ & $4.8 \pm 0.3$ & $\mathrm{~F}=102.004 *$ & $<0.001 *$ \\
\hline \multirow[t]{2}{*}{ Sig. bet. grps. } & \multicolumn{3}{|c|}{$\mathrm{P}_{1}=0.644, \mathrm{p}_{2}<0.00 I^{*}, \mathrm{p}_{3}<0.001 *$} & & \\
\hline & Group I $(n=80)$ & Group II $(n=60)$ & Group III $(n=60)$ & Test of Sig. & $\mathbf{P}$ \\
\hline \multicolumn{6}{|l|}{ Total cholesterol (mg/dl) } \\
\hline Mean \pm SD. & $256.4 \pm 18$ & $205.7 \pm 39.2$ & $170.7 \pm 29.1$ & $\mathrm{~F}=154.058^{*}$ & $<0.001 *$ \\
\hline Sig. bet. grps & \multicolumn{3}{|c|}{$\mathrm{P}_{1}<0.00 \mathrm{I}^{*}, \mathrm{P}_{2}<0.00 \mathrm{I}^{*}, \mathrm{P}_{3}<0.00 \mathrm{I}^{*}$} & & \\
\hline \multicolumn{6}{|l|}{ HDL-C (mg/dl) } \\
\hline Mean \pm SD & $49.2 \pm 10.3$ & $47.8 \pm 10$ & $47.3 \pm 9.6$ & $F=0.660$ & 0.518 \\
\hline \multicolumn{6}{|l|}{ LDL-C (mg/dl) } \\
\hline Mean \pm SD. & $|36| \pm 30.9$. & $129.2 \pm 25.3$ & $93.4 \pm 8.1$ & $\mathrm{~F}=57.257^{*}$ & $<0.001 *$ \\
\hline Sig. bet. grps & \multicolumn{3}{|c|}{$\mathrm{P}_{1}=0.223, \mathrm{P}_{2}<0.00 \mathrm{I}^{*}, \mathrm{P}_{3}<0.00 \mathrm{I}^{*}$} & & \\
\hline
\end{tabular}

(Continued) 
Table I (Continued).

\begin{tabular}{|c|c|c|c|c|c|}
\hline & Group I $(n=80)$ & Group II $(n=60)$ & Group III $(n=60)$ & Test of Sig. & $\mathbf{p}$ \\
\hline Triglycerides (mg/dl) & & & & & \\
\hline Mean \pm SD. & $143.6 \pm 45.7$ & $119.7 \pm 25.6$ & $89.7 \pm 6$ & $\mathrm{~F}=47.729 *$ & $<0.001 *$ \\
\hline Sig. bet. grps & \multicolumn{3}{|c|}{$\mathrm{P}_{1}<0.00 \mathrm{I}^{*}, \mathrm{P}_{2}<0.00 I^{*}, \mathrm{p}_{3}<0.00 I^{*}$} & & \\
\hline Serum creatinine $(\mathrm{mg} / \mathrm{dl})$ & & & & & \\
\hline Mean $\pm S D$ & $2.27 \pm 0.47$ & $0.90 \pm 0.14$ & $0.83 \pm 0.18$ & $\mathrm{~F}=464.784^{*}$ & $<0.001 *$ \\
\hline Sig. bet. grps & \multicolumn{3}{|c|}{$\mathrm{P}_{1}<0.00 I^{*}, \mathrm{p}_{2}<0.00 I^{*}, \mathrm{p}_{3}=0.379$} & & \\
\hline \multicolumn{6}{|l|}{ Serum urea $(\mathbf{m g} / \mathrm{dl})$} \\
\hline Mean \pm SD. & $68.1 \pm 15.2$ & $23.6 \pm 3.1$ & $22.4 \pm 3.6$ & $\mathrm{~F}=494.17 I^{*}$ & $<0.001 *$ \\
\hline Sig. bet. grps & \multicolumn{3}{|c|}{$\mathrm{P}_{1}<0.00 \mathrm{I} * \mathrm{P}_{2}<0.00 \mathrm{I} * \mathrm{P}_{3}=0.807$} & & \\
\hline \multicolumn{6}{|l|}{ ACR (mg/g) } \\
\hline Median (IQR) & $825.0(400.0-869.5)$ & $20.0(17.5-22.0)$ & $18.0(15.0-2 \mid .0)$ & $\mathrm{H}=144.85^{*}$ & $<0.001 *$ \\
\hline Sig. bet. grps & \multicolumn{3}{|c|}{$\mathrm{P}_{1}<0.00 \mathrm{I} * \mathrm{P}_{2}<0.00 \mathrm{I} * \mathrm{P}_{3}=0.247$} & & \\
\hline \multicolumn{6}{|l|}{ eGFR $\left(\mathrm{mL} / \mathrm{min} / 1.73 \mathrm{~m}^{2}\right)$} \\
\hline Mean \pm SD. & $57.31 \pm 8.75$ & $89.70 \pm|2.8|$ & $90.28 \pm 16.03$ & $\mathrm{~F}=163.470^{*}$ & $<0.001 *$ \\
\hline Sig. bet. grps & \multicolumn{3}{|c|}{$\mathrm{P}_{1}<0.00 I^{*}, \mathrm{p}_{2}<0.00 \mathrm{I}^{*}, \mathrm{P}_{3}=0.965$} & & \\
\hline
\end{tabular}

Notes: Group I: Diabetic nephropathy, Group II: Type 2 diabetics without diabetic nephropathy, Group III: Control, $\chi^{2}$ : Chi squared test, F: ANOVA test, H: Kruskal Wallis test, Pairwise comparison between each 2 groups was done using Post Hoc Test (Tukey) *Statistically significant at $p \leq 0.05$.

Abbreviations: DM, diabetes mellitus; BMI, body mass index; SBP, systolic blood pressure; DBP, diastolic blood pressure; FBG, fasting blood glucose; 2 h PPG, 2 hour postprandial glucose; HbAIc, glycated hemoglobin, $\mathrm{PI}_{1}$, P value for comparing between group I and group II; $\mathrm{P}_{2}$, P value for comparing between group I and group III; $\mathrm{P}_{3}$, P value for comparing between group II and group III.

percentage of males was $77.5 \%, 46.7 \%$ and $48.3 \%$ in group I, group II and group III respectively $(\mathrm{p}<0.001)$. There was a significant difference between the three studied groups regarding family history of DM $(\mathrm{p}<0.001)$. When comparing both group I and group II with group III, significantly higher values of BMI $(\mathrm{p}=0.001)$, FBG $(\mathrm{P}=0.001), \mathrm{HbA1c}(\mathrm{p}=0.001)$ and LDL-C $(\mathrm{p}=0.001)$ were found in group I and group II. There was a higher incidence of hypertension in group I than in group II $(\mathrm{p}<$ 0.001). All variables such as smoking, SBP, $2 \mathrm{~h}$ PPG, total cholesterol and triglycerides differed significantly between the three groups. When comparing group I and group II, there was significantly higher DBP in group I $(\mathrm{p}=0.046)$, and no significant difference regarding duration of $\mathrm{DM}$ $(p=0.074), \quad$ BMI $(p=0.328), \quad$ FBG $(p=0.720), \quad H b A 1 c$ $(\mathrm{p}=0.644)$ and LDL-C $(\mathrm{p}=0.223)$. In group $\mathrm{I}$, there was significantly higher DBP $(\mathrm{p}=0.017)$ when compared to group III, while DBP did not significantly differ between group II and group III ( $\mathrm{p}=0.939)$. Regarding kidney function tests, there were significantly higher urea, creatinine, and ACR in group I compared to group II and group III $(p=0.001)$, with no significant difference between group II and group III ( $\mathrm{p}=0.379,0.807,0.247$ respectively). The eGFR showed significantly lower values in group I compared to group II $(\mathrm{p}=0.001)$ and group III $(\mathrm{p}=0.001)$, with no significant difference between group II and group III $(\mathrm{p}=0.965)$.

\section{The Association of EPHX2 Rs75I I4I and CYP2J2 Rs2280275 with the Risk of DN}

Both polymorphisms were in accordance with HardyWeinberg equilibrium ( $>0.05)$. Talking about EPHX2 rs751141 results, there were significant differences in genotype and allele distribution $(\mathrm{p}=0.005$ and $\mathrm{p}<0.001$ respectively) between the three studied groups. In group I, there were significantly higher and lower frequencies of GG and AA genotypes respectively (Table 2). Based on the data, the EPHX2 rs751141 was associated with an increased risk of DN under a dominant model (GG vs $\mathrm{GA}+\mathrm{AA}: \mathrm{OR}=0.375 ; 95 \%$ CI $(0.19-0.75), \mathrm{P}=0.006)$ in unadjusted model and after adjustment for age and sex ( $\mathrm{OR}=0.440 ; 95 \%$ CI (0.21-0.92), $\mathrm{P}=0.029)$, recessive model (AA vs $\mathrm{GG}+\mathrm{GA}: \mathrm{OR}=0.195 ; 95 \%$ CI $(0.05-0.74)$, $\mathrm{P}=0.017$ ), and additive model (GA vs $\mathrm{GG}+\mathrm{AA}$ ): 
Table 2 Prevalence of EPHX2 (Rs75I, I4I) and CYP2J2 (Rs2,280,275) Genotypes and Alleles in the Studied Groups

\begin{tabular}{|c|c|c|c|c|c|}
\hline & Group I $(n=80)$ & Group II $(n=60)$ & Group III $(n=60)$ & $\chi^{\chi}$ & ${ }^{M C} p$ \\
\hline EPHX2 rs75II4I & & & & & \\
\hline $\mathrm{GG}^{\circledR}$ & $56(70 \%)$ & $28(46.7 \%)$ & $26(43.3 \%)$ & $\chi \chi \chi \chi \chi \chi \chi \chi^{*}$ & $0.005^{*}$ \\
\hline GA & $21(26.3 \%)$ & $22(36.7 \%)$ & $23(38.3 \%)$ & & \\
\hline AA & $3(3.8 \%)$ & $10(16.7 \%)$ & II(I8.3\%) & & \\
\hline Sig. bet. grps. & \multicolumn{3}{|c|}{$\chi_{1}^{\chi}=10.483^{*}, \mathrm{p}_{1}=0.005^{*}, \chi_{\chi}^{\chi}=13.047^{*}, \mathrm{p}_{2}=0.00 \mathrm{I}^{*}, \chi_{\chi}^{\chi}=0.144, \mathrm{p}_{3}=0.931$} & & \\
\hline \multicolumn{6}{|l|}{ Allele frequency } \\
\hline$G^{\circledR}$ & $133(83.1 \%)$ & $78(65 \%)$ & $75(62.5 \%)$ & $\chi^{7} \chi^{869} *$ & $<0.001 *$ \\
\hline A & $27(16.9 \%)$ & $42(35 \%)$ & $45(37.5 \%)$ & & \\
\hline Sig. bet. grps. & \multicolumn{3}{|c|}{$\chi_{1}^{\chi}=12.131, \mathrm{p}_{1}<0.00 I^{*}, \chi_{\chi}^{\chi}=15.270^{*}, \mathrm{p}_{2}<0.00 I^{*}, \chi_{\chi}^{\chi}=0.162, \mathrm{p}_{3}=0.687$} & & \\
\hline Dominant & & & & & \\
\hline $\mathrm{GG}^{\circledR}$ & $56(70 \%)$ & $28(46.7 \%)$ & $26(43.3 \%)$ & $\chi \chi \chi x \chi \chi 6^{6 *}$ & $0.002 *$ \\
\hline $\mathrm{GA}+\mathrm{AA}$ & $24(30 \%)$ & $32(53.3)$ & $34(56.7 \%)$ & & \\
\hline \multicolumn{6}{|l|}{ Recessive } \\
\hline$A A^{\circledR}$ & $3(3.8 \%)$ & $10(16.7 \%)$ & II(I8.3\%) & $8 \chi 67 \chi^{*}$ & $0.013^{*}$ \\
\hline$G G+G A$ & $77(96.3 \%)$ & $50(83.3 \%)$ & $49(81.7 \%)$ & & \\
\hline \multicolumn{6}{|l|}{ Additive } \\
\hline AA vs GA & $3 / 21(14.3 \%)$ & $10 / 22(45.5 \%)$ & $1 \mathrm{I} / 23(47.8 \%)$ & 3.369 & 0.186 \\
\hline GG vs GA & $56 / 21(37.5 \%)$ & $28 / 22(78.6 \%)$ & $26 / 23(88.5 \%)$ & $6 \chi \chi \chi \chi \chi^{*}$ & $0.045^{*}$ \\
\hline \multirow[t]{2}{*}{ GA vs $G G+A A$} & $3 / 77(3.9 \%)$ & $10 / 50(20 \%)$ & $11 / 49(22.4 \%)$ & $8 \chi 67 \chi^{*}$ & $0.013^{*}$ \\
\hline & Group I $(n=80)$ & Group II $(n=60)$ & Group III $(n=60)$ & $\chi^{\chi}$ & $\mathbf{P}$ \\
\hline \multicolumn{6}{|l|}{ CYP2J2 rs 2280275} \\
\hline $\mathrm{CC}^{\circledR}$ & $60(75 \%)$ & $47(78.3 \%)$ & $46(76.7 \%)$ & $\chi \chi 4 \chi \chi$ & $M C_{p}=0.997$ \\
\hline $\mathrm{CT}$ & $17(21.3 \%)$ & II(I8.3\%) & $12(20 \%)$ & & \\
\hline $\mathrm{TT}$ & $3(3.8 \%)$ & $2(3.3 \%)$ & $2(3.3 \%)$ & & \\
\hline Sig. bet. grps. & \multicolumn{3}{|c|}{$\chi_{1}^{\chi}=0.298, \mathrm{p}_{1}=0.938, \chi_{\chi}^{\chi}=0.152, \mathrm{p}_{2}=1.000, \chi_{\chi}^{\chi}=0.199, \mathrm{p}_{3}=1.000$} & & \\
\hline \multicolumn{6}{|l|}{ Allele frequency } \\
\hline$C^{\circledR}$ & $137(85.6 \%)$ & $105(87.5 \%)$ & $104(86.7 \%)$ & $\chi x \alpha \chi \chi \alpha$ & 0.900 \\
\hline $\mathrm{T}$ & $23(14.4 \%)$ & $15(12.5 \%)$ & $16(13.3 \%)$ & & \\
\hline Sig. bet. grps. & \multicolumn{3}{|c|}{$\chi^{\chi}{ }_{1}=0.206, \mathrm{p}_{1}=0.650, \chi_{\chi}^{\chi}=0.062, \mathrm{p}_{2}=0.803, \chi_{\chi}^{\chi}=0.037, \mathrm{p}_{3}=0.847$} & & \\
\hline \multicolumn{6}{|l|}{ Dominant } \\
\hline $\mathrm{CC}$ & $60(75 \%)$ & 47(78.3\%) & $46(76.7 \%)$ & 0.213 & 0.899 \\
\hline $\mathrm{CT}+\mathrm{TT}$ & $20(25 \%)$ & $13(21.7 \%)$ & $14(23.3 \%)$ & & \\
\hline \multicolumn{6}{|l|}{ Recessive } \\
\hline $\mathrm{TT}$ & $3(3.8 \%)$ & $2(3.3 \%)$ & $2(3.3 \%)$ & 0.194 & 1.000 \\
\hline $\mathrm{CT}+\mathrm{TT}$ & $77(96.3 \%)$ & $58(96.7 \%)$ & $58(96.7 \%)$ & & \\
\hline \multicolumn{6}{|l|}{ Additive } \\
\hline $\mathrm{CT}$ & $17(21.3 \%)$ & II(I8.3\%) & $12(20 \%)$ & 0.182 & 0.913 \\
\hline $\mathrm{CC}+\mathrm{TT}$ & $63(78.8 \%)$ & $49(81.7 \%)$ & $48(80 \%)$ & & \\
\hline
\end{tabular}

Note: *Statistically significant at $\mathrm{p} \leq 0.05$

Abbreviations: ${ }^{\circledR}$, the reference genotype; MC, Monte Carlo; PI, P value for comparing between group I and group II; $\mathrm{P}_{2}$, P value for comparing betweengroup I and group III; $\mathrm{P}_{3}$, P value for comparing between group II and group III; $\chi_{1}^{\chi}$, Chi squared value for comparing between group I and group II; $\chi_{\chi}^{\chi}$, Chi squared value for comparing between group I and group III; $\chi_{\chi}^{\chi}$, Chi squared value for comparing between group II and group III.

$\mathrm{OR}=0.195 ; 95 \%$ CI $(0.05-0.74), \mathrm{P}=0.017) \quad$ (Table 3). increased risk of $\mathrm{DN}(\mathrm{OR}=0.377 ; 95 \% \mathrm{CI}(0.22-0.66)$, Regarding allele distribution, the significantly decreased $\mathrm{P}=0.001)$ which remained significant after adjustment for frequency of the A allele in group I (16.9\% Table 2) age and sex (OR=0.424; 95\% CI (0.24-0.76), $\mathrm{P}=0.004)$ 
Table 3 Risk Assessment of Diabetic Nephropathy Under the Three Genetic Models for EPHX2 Rs75II4I and CYP2J2 Rs2280275

\begin{tabular}{|c|c|c|c|c|c|c|}
\hline & \multicolumn{2}{|l|}{ Unadjusted } & \multicolumn{2}{|l|}{ Adjusted $^{\mathrm{a}}$} & \multicolumn{2}{|l|}{ Adjusted $^{b}$} \\
\hline & OR $(95 \% \mathrm{CI})$ & $\mathbf{P}$ & OR (95\% CI) & $\mathbf{p}$ & OR (95\% Cl) & $\mathbf{p}$ \\
\hline \multicolumn{7}{|l|}{ EPHX2rs75II4I } \\
\hline Dominant $\left(\mathrm{GG}^{\circledR} \mathrm{vs} \mathrm{GA}+\mathrm{AA}\right)$ & $0.375(0.19-0.75)$ & $0.006 *$ & $0.440(0.21-0.92)$ & $0.029 *$ & $0.681(0.16-2.82)$ & 0.596 \\
\hline Recessive(AA vs $\left.G G+G^{\circledR}{ }^{\circledR}\right)$ & $0.195(0.05-0.74)$ & $0.017^{*}$ & $0.296(0.07-\mid .2 I)$ & 0.091 & $0.284(0.02-3.77)$ & 0.340 \\
\hline Additive $\left(G A\right.$ vs $\left.G G+A A^{\circledR}\right)$ & $0.195(0.05-0.74)$ & $0.017^{*}$ & $0.296(0.07-1.21)$ & 0.091 & $0.284(0.02-3.77)$ & 0.340 \\
\hline $\mathrm{GG}^{\circledR}$ versus GA & $0.477(0.23-1.01)$ & 0.053 & $0.512(0.23-1.12)$ & 0.095 & $0.832(0.18-3.87)$ & 0.815 \\
\hline $\mathrm{GG}^{\circledR}$ versus $\mathrm{AA}$ & $0.150(0.04-0.59)$ & $0.007^{*}$ & $0.165(0.04-0.74)$ & $0.019 *$ & $0.112(0.005-2.28)$ & 0.154 \\
\hline Allele $\left(G^{\circledR}\right.$ versus $\left.A\right)$ & $0.377(0.22-0.66)$ & $0.001 *$ & $0.424(0.24-0.76)$ & $0.004 *$ & $0.447(0.18-1.13)$ & 0.090 \\
\hline \multicolumn{7}{|l|}{ CYP2J2rs2280275 } \\
\hline Dominant $\left(\mathrm{CC}^{\circledR}\right.$ vs $\left.\mathrm{CT}+\mathrm{TT}\right)$ & $1.205(0.54-2.67)$ & 0.646 & $1.160(0.50-2.68)$ & 0.728 & $0.310(0.05-1.97)$ & 0.215 \\
\hline Recessive(TT vs $\mathrm{CT}+\mathrm{TT}^{{ }^{\circledR}}$ ) & $1.130(0.18-6.98)$ & 0.895 & $0.834(0.12-5.61)$ & 0.852 & $1.700(0-67,518.5)$ & 0.922 \\
\hline Additive $\left(\mathrm{CT}\right.$ vs $\left.\mathrm{CC}+\mathrm{TT}^{\circledR}{ }^{\circledR}\right)$ & $1.202(0.52-2.80)$ & 0.670 & $1.228(0.50-2.99)$ & 0.651 & $0.302(0.05-1.92)$ & 0.205 \\
\hline $\mathrm{CC}^{\circledR}$ versus $\mathrm{CT}$ & $1.211(0.52-2.83)$ & 0.659 & $1.221(0.50-2.99)$ & 0.662 & $0.302(0.05-1.93)$ & 0.206 \\
\hline $\mathrm{CC}^{\circledR}$ versus TT & $1.180(0.19-7.32)$ & 0.863 & $0.869(0.13-5.91)$ & 0.886 & I.358(0-309,284.7) & 0.961 \\
\hline Allele $\left(C^{\circledR}\right.$ versus $\left.T\right)$ & I.I75(0.59-2.36) & 0.651 & I.164(0.56-2.42) & 0.684 & $0.450(0.10-2.14)$ & 0.315 \\
\hline
\end{tabular}

Notes: ${ }^{a}$ Adjusted for age and sex, ${ }^{b}$ adjusted for age, sex, BMI, duration of diabetes, hypertension, smoking, total cholesterol, and triglycerides levels. *Statistically significant at $p \leq 0.05$.

Abbreviations: $\mathrm{Cl}$, confidence interval; OR, odds ratio; ${ }^{\circledR}$, the reference genotype.

(Table 3). The genotype GA decreased risk of DN $(\mathrm{OR}=0.477 ; 95 \%$ CI $(0.23-1.01), \mathrm{P}=0.053)$, while AA genotype caused more decreased risk $(\mathrm{OR}=0.150 ; 95 \%$ CI $(0.04-0.59), \mathrm{P}=0.007)$ and $(\mathrm{OR}=0.165 ; 95 \% \mathrm{CI}(0.04$ $0.74), P=0.019$ ) after adjustment for age and sex (Table 3).

The results indicated that the EPHX2 rs751141 might have a significant association with DN risk. However, in the current study, there was no significant association between CYP2J2 rs2280275 and DN risk. The genotypes and allele frequencies of CYP2J2 rs2280275 did not differ significantly between the three studied groups $(\mathrm{p}=0.997$ and $\mathrm{p}=0.900$ respectively). Genotype frequencies of CYP2J2 rs $2280275 \mathrm{CC}$, CT, and TT were $75 \%, 21.3 \%$, and $3.8 \%$ in group I, $78.3 \%, 18.3 \%$, and $3.3 \%$ in group II, and $76.7 \%, 20 \%$, and $3.3 \%$ in group III respectively (Table 2).

Meanwhile, we statistically analyzed the association of EPHX2 rs751141 genotypes and different parameters in DN group. Due to the small number of homozygous variant carriers (AA), both homozygous (AA) and heterozygous (GA) variants were gathered in one group for statistical reasons. $(\mathrm{GA}+\mathrm{AA})$ patients showed significantly lower values of BMI $(p=0.031)$, serum creatinine $(p<$ $0.001), A C R(p<0.001)$ and a significantly higher eGFR $(\mathrm{p}<0.001)$. However, no significant difference was observed between GG patients and (GA+AA) patients regarding age, sex, duration of DM, smoking, hypertension, SBP, DBP, FBG, 2h PPG, HbA1c, lipid panel and serum urea (Table 4).

Analysis of the relation of EPHX2 rs751141 with clinical and biochemical parameters within T2DM without DN (group II), revealed significant differences regarding age $(p=0.023)$, sex $(p=0.041)$, duration of diabetes $(p=0.012)$, and BMI $(p=0.003)$, while other parameters showed non-significant differences (Table 5).

Regarding CYP2J2 rs2280275, diabetic nephropathy patients with $\mathrm{CC}$ genotypes exhibited significantly higher values of creatinine $(p=0.006)$, higher ACR $(p=0.003)$ and lower eGFR $(\mathrm{p}<0.001)$ in comparison to patients with CT +TT genotypes (Table 6). Within T2DM without DN (group II), there were significant differences between CC patients and CT+TT patients regarding age $(\mathrm{p}=0.049)$, duration of diabetes $(\mathrm{p}=0.007)$, BMI $(\mathrm{p}=0.001)$, smoking $(\mathrm{p}=0.028)$ and total cholesterol $(\mathrm{p}=0.002)$ (Table 7).

\section{Discussion}

The restricted EETs' production in the vascular endothelial and renal epithelial cells lines up with the natural activities and commitment of EETs toward cardiovascular and renal health. ${ }^{31,32}$ So, genetic variations in genes controlling synthesis and degradation of EETs may affect renal functions.

The present study observed significant association of EPHX2 rs751141 with DN among T2DM patients. We found significantly higher frequencies of GG genotype 
Table 4 Association of EPHX2 (Rs75II4I) Genotypes with Different Parameters in DN Patients

\begin{tabular}{|c|c|c|c|c|}
\hline & \multicolumn{2}{|c|}{ EPHX2 rs75II4I } & \multirow[t]{2}{*}{ Test of Sig. } & \multirow[t]{2}{*}{$\mathbf{p}$} \\
\hline & GG $(n=56)$ & GA+AA $(n=24)$ & & \\
\hline \multicolumn{5}{|l|}{ Sex n (\%) } \\
\hline Male & $44(78.6 \%)$ & $18(75 \%)$ & $\chi^{2}=0.123$ & 0.726 \\
\hline Female & $12(21.4 \%)$ & $6(25 \%)$ & & \\
\hline \multicolumn{5}{|l|}{ Age (years) } \\
\hline Mean \pm SD. & $58.9 \pm 7.5$ & $57 \pm 10.6$ & $\mathrm{t}=0.804$ & 0.427 \\
\hline Family history of DM $n$ (\%) & $48(85.7 \%)$ & $20(83.3 \%)$ & $\chi^{2}=89.940 *$ & $<0.001 *$ \\
\hline \multicolumn{5}{|l|}{ Duration of DM (years) } \\
\hline Mean \pm SD. & $13.6 \pm 3.6$ & $13 \pm 4.5$ & $\mathrm{t}=0.598$ & 0.552 \\
\hline \multicolumn{5}{|l|}{ BMI $\left(\mathrm{kg} / \mathbf{m}^{2}\right)$} \\
\hline Mean \pm SD. & $25.6 \pm 2.1$ & $24.5 \pm 1.8$ & $\mathrm{t}=2.191 *$ & $0.03 I^{*}$ \\
\hline Current smokers (\%) & $26(46.4 \%)$ & $8(33.3 \%)$ & & \\
\hline History of hypertension (\%) & $29(51.8 \%)$ & $16(66.7 \%)$ & $\chi^{2}=1.512$ & 0.219 \\
\hline \multicolumn{5}{|l|}{ SBP (mmHg) } \\
\hline Mean \pm SD & $127.7 \pm 8$ & $128.3 \pm 9.6$ & $\mathrm{t}=0.266$ & 0.791 \\
\hline \multicolumn{5}{|l|}{ DBP (mmHg) } \\
\hline Mean \pm SD. & $79.5 \pm 6.3$ & $78.8 \pm 7$ & $\mathrm{t}=0.495$ & 0.622 \\
\hline \multicolumn{5}{|l|}{ FBG (mg/dl) } \\
\hline Mean \pm SD. & $196.4 \pm 66.6$ & $195.4 \pm 76.8$ & $\mathrm{t}=0.063$ & 0.950 \\
\hline \multicolumn{5}{|l|}{2 h PPG (mg/dl) } \\
\hline Mean \pm SD. & $314.8 \pm 72.3$ & $305.2 \pm 88.7$ & $\mathrm{t}=0.47 \mathrm{I}$ & 0.640 \\
\hline \multicolumn{5}{|l|}{ HbAlc (\%) } \\
\hline Mean \pm SD. & $8.6 \pm 1.7$ & $8.4 \pm 2.2$ & $\mathrm{t}=0.344$ & 0.732 \\
\hline \multicolumn{5}{|l|}{ Total cholesterol (mg/dl) } \\
\hline Mean \pm SD & $256 \pm 17.5$ & $257.3 \pm 19.6$ & $\mathrm{t}=0.305$ & 0.761 \\
\hline \multicolumn{5}{|l|}{ HDL-C (mg/dl) } \\
\hline Mean \pm SD. & $49.4 \pm 10.8$ & $48.6 \pm 9.3$ & $\mathrm{t}=0.326$ & 0.745 \\
\hline \multicolumn{5}{|l|}{ LDL-C (mg/dl) } \\
\hline Mean \pm SD. & $134.7 \pm 32.6$ & $139.4 \pm 26.8$ & $\mathrm{t}=0.616$ & 0.539 \\
\hline \multicolumn{5}{|l|}{ Triglycerides (mg/dl) } \\
\hline Mean \pm SD. & $\mid 49.1 \pm 48.6$ & $130.8 \pm 35.6$ & $\mathrm{t}=1.656$ & 0.102 \\
\hline \multicolumn{5}{|l|}{ Serum creatinine (mg/dl) } \\
\hline Mean \pm SD & $2.5 \pm 0.4$ & $1.8 \pm 0.2$ & $\mathrm{t}=10.283$ & $<0.001 *$ \\
\hline \multicolumn{5}{|l|}{ Serum urea $(\mathbf{m g} / \mathrm{dl})$} \\
\hline Mean \pm SD. & $69 \pm 15$ & $66.1 \pm 15.7$ & $\mathrm{t}=0.791$ & 0.431 \\
\hline \multicolumn{5}{|l|}{ ACR (mg/g) } \\
\hline Median (IQR) & $852.5(82 I-9 I I)$ & $346.5(302-391)$ & $U=0.0^{*}$ & $<0.001 *$ \\
\hline eGFR $\left(\mathrm{mL} / \mathrm{min} / 1.73 \mathrm{~m}^{2}\right)$ & & & & \\
\hline
\end{tabular}

Notes: Mean \pm SD. $52.2 \pm 4.569 .1 \pm 2.5 \mathrm{t}=21.445 *<0.00$ I*, *statistically significant at $\mathrm{p} \leq 0.05$

Abbreviations: $U$, Mann Whitney test; $t$, Student's $t$-test; $\chi^{2}$, Chi squared test. 
Table 5 Association of EPHX2 Rs75II4I Genotypes with Different Parameters in T2DM Patients Without Nephropathy

\begin{tabular}{|c|c|c|c|c|}
\hline & \multicolumn{2}{|c|}{ EPHX2 rs 75II4I } & \multirow[t]{2}{*}{ Test of Sig. } & \multirow[t]{2}{*}{$\mathbf{p}$} \\
\hline & GG $(n=28)$ & GA+AA $(n=32)$ & & \\
\hline \multicolumn{5}{|l|}{ Sex } \\
\hline Male & $17(60.7 \%)$ & $\mathrm{II}(34.4 \%)$ & $\chi^{2}=4.163^{*}$ & $0.041 *$ \\
\hline Female & II(39.3\%) & $21(65.6 \%)$ & & \\
\hline \multicolumn{5}{|l|}{ Age (years) } \\
\hline Mean \pm SD. & $54.1 \pm 8.9$ & $59.6 \pm 9.1$ & $\mathrm{t}=2.338^{*}$ & $0.023 *$ \\
\hline Family history of DM & $18(64.3 \%)$ & $27(84.4 \%)$ & $\chi^{2}=3.214$ & 0.073 \\
\hline \multicolumn{5}{|l|}{ Duration of DM (years) } \\
\hline Mean \pm SD. & $13.0 \pm 4.9$ & $16.7 \pm 5.9$ & $\mathrm{t}=2.583^{*}$ & $0.012 *$ \\
\hline \multicolumn{5}{|l|}{ BMI $\left(\mathrm{kg} / \mathrm{m}^{2}\right)$} \\
\hline Mean \pm SD. & $25.0 \pm 1.6$ & $26.3 \pm 1.6$ & $\mathrm{t}=3.150^{*}$ & $0.003 *$ \\
\hline Current smokers (\%) & II(39.3\%) & $19(59.4 \%)$ & $\chi^{2}=2.411$ & 0.121 \\
\hline History of hypertension (\%) & $9(32.1 \%)$ & $8(25 \%)$ & $\chi^{2}=0.375$ & 0.540 \\
\hline \multicolumn{5}{|l|}{ SBP (mmHg) } \\
\hline Mean \pm SD. & $121.5 \pm 12.7$ & $122.8 \pm 9.2$ & $\mathrm{t}=0.442$ & 0.660 \\
\hline \multicolumn{5}{|l|}{ DBP (mmHg) } \\
\hline Mean \pm SD & $75.1 \pm 8.4$ & $78.0 \pm 6.8$ & $\mathrm{t}=1.439$ & 0.155 \\
\hline \multicolumn{5}{|l|}{ FBG (mg/dl) } \\
\hline Mean \pm SD. & $206.5 \pm 60.5$ & $200.8 \pm 65.1$ & $\mathrm{t}=0.349$ & 0.728 \\
\hline \multicolumn{5}{|l|}{ 2hr PPG (mg/dl) } \\
\hline Mean \pm SD. & $292.4 \pm 60.1$ & $281.6 \pm 48.6$ & $\mathrm{t}=0.767$ & 0.446 \\
\hline \multicolumn{5}{|l|}{ HbAlc (\%) } \\
\hline Mean \pm SD. & $9.1 \pm 2.4$ & $8.5 \pm 2.2$ & $t=0.901$ & 0.371 \\
\hline \multicolumn{5}{|l|}{ Total cholesterol (mg/dl) } \\
\hline Mean \pm SD. & $200.1 \pm 41.7$ & $210.5 \pm 36.8$ & $\mathrm{t}=1.022$ & 0.311 \\
\hline \multicolumn{5}{|l|}{ HDL-C (mg/dl) } \\
\hline Mean \pm SD. & $49.4 \pm 9.6$ & $46.4 \pm 10.2$ & $\mathrm{t}=\mathrm{I} .187$ & 0.240 \\
\hline \multicolumn{5}{|l|}{ LDL-C (mg/dl) } \\
\hline Mean \pm SD. & $123.2 \pm 25.7$ & $134.4 \pm 24.2$ & $\mathrm{t}=\mathrm{I} .740$ & 0.087 \\
\hline \multicolumn{5}{|l|}{ Triglycerides (mg/dl) } \\
\hline Mean \pm SD. & $116.6 \pm 27.2$ & $122.4 \pm 24.2$ & $\mathrm{t}=0.874$ & 0.386 \\
\hline \multicolumn{5}{|l|}{ Serum creatinine $(\mathrm{mg} / \mathrm{dl})$} \\
\hline Mean \pm SD. & $0.9 \pm 0.1$ & $0.9 \pm 0.2$ & $\mathrm{t}=0.5 \mathrm{I} 5$ & 0.609 \\
\hline \multicolumn{5}{|l|}{ Serum urea $(\mathrm{mg} / \mathrm{dl})$} \\
\hline Mean \pm SD. & $23.9 \pm 3.5$ & $23.2 \pm 2.8$ & $\mathrm{t}=0.872$ & 0.387 \\
\hline \multicolumn{5}{|l|}{ ACR (mg/g) } \\
\hline Median (IQR) & $19.5(\mid 7.5-22.5)$ & $20(|7.5-2| .5)$ & $U=408.5$ & 0.557 \\
\hline \multicolumn{5}{|l|}{ eGFR (mL/min/l.73m2) } \\
\hline Mean \pm SD. & $88.6 \pm 15.5$ & $90.7 \pm 10.1$ & $\mathrm{t}=0.598$ & 0.553 \\
\hline
\end{tabular}

Note: *Statistically significant at $\mathrm{p} \leq 0.05$.

Abbreviations: $U$, Mann Whitney test; $t$, Student's $t$-test; $\chi^{2}$, Chi squared test. 
Table 6 Relation Between CYP2J2 Rs2280275 and Different Parameters in DN

\begin{tabular}{|c|c|c|c|c|}
\hline & \multicolumn{2}{|c|}{ CYP2J2rs2280275 } & \multirow[t]{2}{*}{ Test of Sig. } & \multirow[t]{2}{*}{$\mathbf{p}$} \\
\hline & $C C(n=60)$ & $C T+T T(n=20)$ & & \\
\hline \multicolumn{5}{|l|}{ Sex n (\%) } \\
\hline Male & $45(75 \%)$ & $17(85 \%)$ & $\chi^{2}=0.860$ & 0.538 \\
\hline Female & $15(25 \%)$ & $3(15 \%)$ & & \\
\hline \multicolumn{5}{|l|}{ Age (years) } \\
\hline Mean \pm SD. & $59 \pm 7.3$ & $56.3 \pm 11.3$ & $\mathrm{t}=1.004$ & 0.325 \\
\hline Family history of DM n (\%) & $51(85 \%)$ & 19(95\%) & $\chi^{2}=|.37|$ & 0.437 \\
\hline \multicolumn{5}{|l|}{ Duration of DM (years) } \\
\hline Mean \pm SD. & $13.7 \pm 3.5$ & $12.5 \pm 4.9$ & $\mathrm{t}=\mathrm{I} .262$ & 0.211 \\
\hline \multicolumn{5}{|l|}{ BMI $\left(\mathrm{kg} / \mathrm{m}^{2}\right)$} \\
\hline Mean \pm SD. & $25.4 \pm 2$ & $24.7 \pm 2.1$ & $\mathrm{t}=\mathrm{I} .457$ & 0.149 \\
\hline Current smokers (\%) & $26(43.3 \%)$ & $8(40 \%)$ & $\chi^{2}=0.068$ & 0.794 \\
\hline History of hypertension (\%) & $32(53.3 \%)$ & $13(65 \%)$ & $\chi^{2}=0.830$ & 0.362 \\
\hline \multicolumn{5}{|l|}{ SBP (mmHg) } \\
\hline Mean \pm SD & $128.2 \pm 8.4$ & $127 \pm 8.7$ & $\mathrm{t}=0.554$ & $0.58 \mathrm{I}$ \\
\hline \multicolumn{5}{|l|}{ DBP (mmHg) } \\
\hline Mean \pm SD. & $79.6 \pm 6.5$ & $78.5 \pm 6.5$ & $\mathrm{t}=0.635$ & 0.527 \\
\hline \multicolumn{5}{|l|}{ FBG (mg/dl) } \\
\hline Mean \pm SD. & $193.1 \pm 65.8$ & $205.4 \pm 80$ & $\mathrm{t}=0.685$ & 0.495 \\
\hline \multicolumn{5}{|l|}{ 2hr PPG (mg/dl) } \\
\hline Mean \pm SD. & $307.9 \pm 73.8$ & $324.1 \pm 87.5$ & $\mathrm{t}=0.812$ & 0.419 \\
\hline \multicolumn{5}{|l|}{ HbAlc (\%) } \\
\hline Mean \pm SD. & $8.4 \pm 1.6$ & $9 \pm 2.4$ & $\mathrm{t}=1.176$ & 0.732 \\
\hline \multicolumn{5}{|l|}{ Total cholesterol (mg/dl) } \\
\hline Mean \pm SD & $254.9 \pm 18.3$ & $261 \pm 16.8$ & $\mathrm{t}=1.313$ & 0.193 \\
\hline \multicolumn{5}{|l|}{ HDL-C (mg/dl) } \\
\hline Mean \pm SD. & $50 \pm 10.7$ & $46.7 \pm 9$ & $\mathrm{t}=1.260$ & 0.211 \\
\hline \multicolumn{5}{|l|}{ LDL-C (mg/dl) } \\
\hline Mean \pm SD. & $134.4 \pm 32$ & $|4| .2 \pm 27.3$ & $\mathrm{t}=0.84 \mathrm{I}$ & 0.403 \\
\hline \multicolumn{5}{|l|}{ Triglycerides (mg/dl) } \\
\hline Mean \pm SD. & $|4| .9 \pm 46.5$ & $148.8 \pm 44$ & $\mathrm{t}=0.578$ & 0.565 \\
\hline \multicolumn{5}{|l|}{ Serum creatinine (mg/dl) } \\
\hline Mean \pm SD & $2.4 \pm 0.5$ & $2 \pm 0.4$ & $\mathrm{t}=2.826^{*}$ & $0.006^{*}$ \\
\hline \multicolumn{5}{|l|}{ Serum urea $(\mathbf{m g} / \mathrm{dl})$} \\
\hline Mean \pm SD. & $67.8 \pm 15.3$ & $69.2 \pm 15$ & $\mathrm{t}=0.343$ & 0.733 \\
\hline \multicolumn{5}{|l|}{ ACR (mg/g) } \\
\hline Median (IQR) & $839(793-886.5)$ & $390(330-806)$ & $U=328.5^{*}$ & $0.003^{*}$ \\
\hline \multicolumn{5}{|l|}{ eGFR $\left(\mathrm{mL} / \mathrm{min} / 1.73 \mathrm{~m}^{2}\right)$} \\
\hline Mean \pm SD. & $54.8 \pm 7.5$ & $64.8 \pm 7.9$ & $\mathrm{t}=5.095^{*}$ & $<0.001 *$ \\
\hline
\end{tabular}

Note: *Statistically significant at $\mathrm{p} \leq 0.05$.

Abbreviations: $\chi^{2}$, Chi squared test; $t$, Student's $t$-test; $U$, Mann Whitney test. 
Table 7 Association of CYP2J2 Rs2280275 with Different Parameters in T2DM Patients Without Nephropathy

\begin{tabular}{|c|c|c|c|c|}
\hline & \multicolumn{2}{|c|}{ CYP2J2 rs2280275 } & \multirow[t]{2}{*}{ Test of Sig. } & \multirow[t]{2}{*}{$\mathbf{p}$} \\
\hline & $C C(n=47)$ & $C T+T T(n=13)$ & & \\
\hline \multicolumn{5}{|l|}{ Sex } \\
\hline Male & $23(48.9 \%)$ & $5(38.5 \%)$ & $\chi^{2}=0.449$ & 0.503 \\
\hline Female & $24(51.1 \%)$ & $8(61.5 \%)$ & & \\
\hline \multicolumn{5}{|l|}{ Age (years) } \\
\hline Mean \pm SD. & $55.8 \pm 9.6$ & $61.5 \pm 7.1$ & $\mathrm{t}=2.007^{*}$ & $0.049 *$ \\
\hline Family history of DM (\%) & $35(74.5 \%)$ & $10(76.9 \%)$ & $\chi^{2}=0.033$ & 1.000 \\
\hline \multicolumn{5}{|l|}{ Duration of DM (years) } \\
\hline Mean \pm SD. & $13.9 \pm 5.5$ & $18.7 \pm 5.2$ & $\mathrm{t}=2.814^{*}$ & $0.007 *$ \\
\hline \multicolumn{5}{|l|}{ BMI $\left(\mathrm{kg} / \mathrm{m}^{2}\right)$} \\
\hline Mean \pm SD & $25.4 \pm 1.8$ & $26.7 \pm 0.9$ & $\mathrm{t}=3.598^{*}$ & $0.001 *$ \\
\hline Current smokers (\%) & $20(42.6 \%)$ & $10(76.9 \%)$ & $\chi^{2}=4.812^{*}$ & $0.028 *$ \\
\hline History of hypertension (\%) & $14(29.8 \%)$ & $3(23.1 \%)$ & $\chi^{2}=0.226$ & 0.740 \\
\hline \multicolumn{5}{|l|}{ SBP (mmHg) } \\
\hline Mean \pm SD & $122.4 \pm 11.8$ & $121.5 \pm 6.9$ & $\mathrm{t}=0.337$ & 0.738 \\
\hline \multicolumn{5}{|l|}{ DBP (mmHg) } \\
\hline Mean \pm SD. & $76.0 \pm 8.2$ & $78.8 \pm 4.6$ & $\mathrm{t}=1.594$ & 0.120 \\
\hline \multicolumn{5}{|l|}{ FBG (mg/dl) } \\
\hline Mean \pm SD. & $205.7 \pm 61.5$ & $195.7 \pm 68.1$ & $t=0.505$ & 0.615 \\
\hline \multicolumn{5}{|l|}{ 2hr PPG (mg/dl) } \\
\hline Mean \pm SD. & $287.2 \pm 56.7$ & $284.8 \pm 45.2$ & $\mathrm{t}=0.141$ & 0.889 \\
\hline \multicolumn{5}{|l|}{ HbAlc (\%) } \\
\hline Mean \pm SD. & $8.9 \pm 2.2$ & $8.4 \pm 2.6$ & $t=0.732$ & 0.467 \\
\hline \multicolumn{5}{|l|}{ Total cholesterol (mg/dl) } \\
\hline Mean \pm SD. & $200.0 \pm 41.5$ & $226.2 \pm 18.7$ & $\mathrm{t}=3.228^{*}$ & $0.002 *$ \\
\hline \multicolumn{5}{|l|}{ HDL-C (mg/dl) } \\
\hline Mean \pm SD. & $48.7 \pm 10.2$ & $44.5 \pm 8.9$ & $\mathrm{t}=1.34 \mid$ & 0.185 \\
\hline \multicolumn{5}{|l|}{ LDL-C (mg/dl) } \\
\hline Mean \pm SD. & $128.7 \pm 24.7$ & $|3| . \mid \pm 28.7$ & $\mathrm{t}=0.299$ & 0.766 \\
\hline \multicolumn{5}{|l|}{ Triglycerides (mg/dl) } \\
\hline Mean \pm SD. & $118.0 \pm 25.4$ & $125.9 \pm 26.4$ & $\mathrm{t}=0.986$ & 0.328 \\
\hline \multicolumn{5}{|l|}{ Serum creatinine (mg/dl) } \\
\hline Mean \pm SD. & $0.9 \pm 0.1$ & $0.9 \pm 0.1$ & $\mathrm{t}=1.613$ & 0.112 \\
\hline \multicolumn{5}{|l|}{ Serum urea $(\mathrm{mg} / \mathrm{dl})$} \\
\hline Mean \pm SD & $23.7 \pm 3.4$ & $23.0 \pm 2.1$ & $\mathrm{t}=0.925$ & 0.362 \\
\hline \multicolumn{5}{|l|}{ ACR (mg/g) } \\
\hline Median (IQR) & $20(17-22)$ & $19(|8-2|)$ & $U=294.50$ & 0.843 \\
\hline \multicolumn{5}{|l|}{ eGFR (mL/min/1.73m2) } \\
\hline Mean \pm SD. & $88.6 \pm 13.4$ & $93.5 \pm 10.1$ & $\mathrm{t}=\mathrm{I} .226$ & 0.225 \\
\hline
\end{tabular}

Note: *Statistically significant at $\mathrm{p} \leq 0.05$.

Abbreviations: $U$, Mann Whitney test; $t$, Student's $t$-test; $\chi^{2}$, Chi squared test. 
and $\mathrm{G}$ allele and significantly lower frequencies of $\mathrm{AA}$ genotype and $\mathrm{A}$ allele in $\mathrm{DN}$ patients in comparison to T2DM without DN patients. The presence of the A allele reduced the odds of $\mathrm{DN}(\mathrm{OR}=0.377)$. Such differences may indicate that carriers of the risk-free $\mathrm{A}$ allele are less susceptible to developing DN. This significant association was present under the three genetic models (dominant, recessive and additive).

The EPHX2 rs751141 polymorphism involves substitution of $\mathrm{A}$ instead of $\mathrm{G}$ in exon 8 of the gene encoding $\mathrm{sEH}$. This nucleotide substitution leads to amino acid substitution from arginine to glutamine at codon 287 (R287Q). The A allele results in a form of sEH enzyme with lower EETs hydrolytic capability resulting in higher levels of EETs. ${ }^{33}$ Yi et al, documented lower EETs level in GG patients in comparison to GA and AA patients that was associated with development of early neurological deterioration in Chinese patients with acute minor ischemic stroke. $^{20}$ A previous study revealed that lower levels of EETs in glomeruli of the kidney are involved in glomerular deterioration in the early stage of DN. ${ }^{34}$ Now it turns out that the renoprotective actions of persistently higher EETs levels in diabetic patients with the rs751141 A allele might be one of the reasons of being less susceptible to DN. ${ }^{19}$

Endothelial impairment is a hallmark of cardiovascular and renal diseases. ${ }^{21}$ EETs maintain endothelial health. ${ }^{22}$ So, EPHX2 rs751141 might be implicated in progression of DN. As previously shown, the A allele will lead to slow degradation of EETs ${ }^{19,35}$ and consequently, endothelial protection and resistance to glomerular injury. In line with this, our DN patients with the A allele showed significantly lower serum creatinine, lower ACR and higher eGFR in comparison to levels in GG patients. This means that the A allele might counteract DN progression and preserve, to some extent, kidney function among these patients.

In the following paragraphs, we will list the results of several studies that unquestionably support the protective effect of the A allele of EPHX2 rs751141 in vascular associated diseases.

Ma et al, in a study performed on Chinese T2DM patients, recorded significantly different distribution of EPHX2 rs751141 genotypes and alleles between DM patients with or without DN, with lower frequency of the A allele $(20.94 \%)$ in DN patients. They also demonstrated that the AA genotype decreased risk of DN by $73 \%$ after adjustment for established DN risk factors. ${ }^{23}$
The study done by Lee et al, revealed a significant effect of EPHX2 rs751141 on progression of IgA nephropathy $(\operatorname{IgAN})$ in Korean population. They noticed better kidney survival in IgAN patients with the A allele, remarkable kidney vascular damage in patients with the $G$ allele and higher in vivo activity of sEH in patients carrying the G allele. ${ }^{22}$ They assumed that the A allele decreased sEH activity in IgAN patients affecting intrarenal blood pressure preserving kidney function. Moreover, they found that patients with GG genotype showed significantly lower eGFR and higher systolic blood pressure than other groups (GA/AA).

Our data additionally bolsters the results of past reports that the A allele of EPHX2 rs751141 confers significantly lower risk of ischemic stroke. ${ }^{19,36}$ Donelly et al attributed the reason behind better outcomes in the A allele patients with aneurysmal subarachnoid hemorrhage to the fact that the EPHX2 287Gln variant is a missense SNP accompanied by diminished EETs degradation and ischemic cell death. $^{37}$

Consistently, several experimental studies indicated that genetic inhibition of sEH protects the kidney against diabetic injury, as evident by decreased creatinine, blood urea nitrogen and urinary albumin excretion. ${ }^{5,16,38}$

In contrast, Lee et al, in their study on 204 kidney transplant donor-recipient population, found no significant association between allograft dysfunction and EPHX2 rs751141 GG genotype. The explanation given was the lack of prolonged follow-up. ${ }^{21}$ Moreover, Gervasini et al, stated that EPHX2 rs751141 did not affect serum creatinine and eGFR in Caucasian renal transplant recipients and deceased donors. ${ }^{6}$

CYP2J2 is one of the key enzymes of epoxygenase pathway of AA. ${ }^{9}$ The anti-hypoxic, anti-inflammatory, xenobiotic detoxification ability, and EETs' generation qualify CYP2J2 protein as a strong candidate for vascular protective lipid metabolizingepoxygenase. ${ }^{39}$

A previous study confirmed the significant association of CYP2J2 rs2280275 with coronary artery disease in a male Uygur population in China, indicating its protective role in vascular endothelium. ${ }^{13}$ Our results did not support what they found, as this study revealed no significant difference in genotype and allele distributions of CYP2J2rs2280275 between DN patients, T2DM patients, and healthy subjects. Based on this evidence, CYP2J2 rs2280275 is probably not involved in genetic susceptibility to DN. However, the relatively small sample size, ethnic and geographic elements 
may explain these findings, so further larger studies are needed.

It is worth noting that our study is the first in terms of examining the CYP2J2 rs2280275 in DN. Moreover, the significant association of EPHX2 rs751141 with creatinine, ACR and eGFR is a novel finding. However, there are some limitations. First, the relatively small sample size may result in lack of association between CYP2J2 rs2280275 and DN. Second, this study did not determine the effect of other functional SNPs in CYP2J2. Thus, further studies on a larger set of SNPs could be constructed. Finally, extensive research is required to elucidate the mechanism of the $E P H X 2$ rs751141 A allele to preserve kidney function.

\section{Conclusion}

No association was found between CYP2J2 rs2280275 and DN susceptibility among Egyptian T2DM patients. In contrast, our study revealed that the EPHX2 rs751141 might be considered as one of the genetic determinants of DN. In fact, it has been shown that the EPHX2 rs751141 A allele is linked not only to protection against $\mathrm{DN}$, but also to reduced disease progression.

\section{Funding}

This article was not funded.

\section{Disclosure}

The authors declare that they have no conflicts of interest.

\section{References}

1. Wei L, Xiao Y, Li L, et al. The susceptibility genes in diabetic nephropathy. Kidney Dis. 2018;4(4):226-237. doi:10.1159/000492633

2. Hill CJ, Cardwell CR, Patterson CC, et al. Chronic kidney disease and diabetes in the national health service: a cross-sectional survey of the U.K. national diabetes audit. Diabet Med. 2014;31(4):448-454. doi:10.1111/dme.12312

3. Gurley SB, Ghosh S, Johnson SA, et al. Inflammation and immunity pathways regulate genetic susceptibility to diabetic nephropathy. Diabetes. 2018;67(10):2096-2106. doi:10.2337/db17-1323

4. Shao J, Wang H, Yuan G, et al. Involvement of the arachidonic acid cytochrome $\mathrm{P} 450$ epoxygenase pathway in the proliferation and invasion of human multiple myeloma cells. Peer J. 2016;4:e1925. doi:10.7717/peerj.1925

5. Chen G, Xu R, Wang Y, et al. Genetic disruption of soluble epoxide hydrolase is protective against streptozotocin-induced diabetic nephropathy. Am J Physiol Endocrinol Metab. 2012;303(5):E563-E575. doi:10.1152/ajpendo.00591.2011

6. Gervasini G, García-Cerrada M, Coto E, et al. A 3'-UTR polymorphism in soluble epoxide hydrolase gene is associated with acute rejection in renal transplant recipients. PLoS One. 2015;10(7):e0133563. doi:10.1371/journal.pone. 0133563
7. Enayetallah AE, French RA, Thibodeau MS, et al. Distribution of soluble epoxide hydrolase and of cytochrome P450 2C8, 2C9, and 2J2 in human tissues. J Histochem Cytochem. 2004;52(4):447-454. doi: $10.1177 / 002215540405200403$

8. Hye Khan MA, Hwang SH, Sharma A, et al. A dual COX-2/sEH inhibitor improves the metabolic profile and reduces kidney injury in Zucker diabetic fatty rat. Prostaglandins Other Lipid Mediat. 2016;125:40-47. doi:10.1016/j.prostaglandins.2016.07.003

9. Nelson DR. The cytochrome p450 homepage. Hum Genomics. 2009;4(1):59-65. doi:10.1186/1479-7364-4-1-59

10. Aliwarga T, Evangelista EA, Sotoodehnia N, et al. Regulation of CYP2J2 and EET levels in cardiac disease and diabetes. Int $J \mathrm{Mol}$ Sci. 2018;19(7):1916. doi:10.3390/ijms 19071916

11. King LM, Ma J, Srettabunjong S, et al. Cloning of CYP2J2 gene and identification of functional polymorphisms. Mol Pharmacol. 2002;61 (4):840-852. doi:10.1124/mol.61.4.840

12. Spiecker M, Darius H, Hankeln T, et al. Risk of coronary artery disease associated with polymorphism of the cytochrome P450 epoxygenase CYP2J2. Circulation. 2004;110(15):2132-2136. doi:10. 1161/01.CIR.0000143832.91812.60

13. Zhu Q, Fu Z, Ma Y, et al. A novel polymorphism of the CYP2J2 gene is associated with coronary artery disease in Uygur population in China. Clin Biochem. 2013;46(12):1047-1054. doi:10.1016/j. clinbiochem.2013.05.003

14. Polonikov AV, Ponomarenko IV, Bykanova MA, et al. A comprehensive study revealed SNP-SNP interactions and a sex-dependent relationship between polymorphisms of the CYP2J2 gene and hypertension risk. Hypertens Res. 2019;42(2):257-272. doi:10.1038/ s41440-018-0142-1

15. Abdu E, Bruun DA, Yang D, et al. Epoxyeicosatrienoic acids enhance axonal growth in primary sensory and cortical neuronal cell cultures. J Neurochem. 2011;117(4):632-642. doi:10.1111/ j.1471-4159.2010.07139.x

16. Elmarakby AA, Faulkner J, Al-Shabrawey M, et al. Deletion of soluble epoxide hydrolase gene improves renal endothelial function and reduces renal inflammation and injury in streptozotocin-induced type 1 diabetes. Am J Physiol RegulIntegr Comp Physiol. 2011;301(5):R1307-R1317. doi:10.1152/ajpregu.00759. 2010

17. Liu JY. Inhibition of Soluble Epoxide Hydrolase for Renal Health. Front Pharmacol. 2019;9:1551. doi:10.3389/fphar.2018.01551

18. Larsson C, White I, Johansson C, et al. Localization of the human soluble epoxide hydrolase gene (EPHX2) to chromosomal region 8p21-p12. Hum Genet. 1995;95(3):356-358. doi:10.1007/bf002 25209

19. Przybyla-Zawislak BD, Srivastava PK, Vazquez-Matias J, et al. Polymorphisms in human soluble epoxide hydrolase. Mol Pharmacol. 2003;64(2):482-490. doi:10.1124/mol.64.2.482

20. Yi X, Lin J, Li J, et al. Epoxyeicosatrienoic acids are mediated by EPHX2 variants and may be a predictor of early neurological deterioration in acute minor ischemic stroke. $J$ Atheroscler Thromb. 2017;24(12):1258-1266. doi:10.5551/jat.41145

21. Lee SH, Lee J, Cha R, et al. Genetic variations in soluble epoxide hydrolase and graft function in kidney transplantation. Transplant Proc. 2008;40(5):1353-1356. doi:10.1016/j.transproceed.2008. 03.137

22. Lee JP, Yang SH, Kim DK, et al. In vivo activity of epoxide hydrolase according to sequence variation affects the progression of human IgA nephropathy. Am J Physiol Renal Physiol. 2011;300(6):F1283F1290. doi:10.1152/ajprenal.00733.2010

23. Ma L, Yan M, Kong X, et al. Association of EPHX2 R287Q polymorphism with diabetic nephropathy in chinese type 2 diabetic patients. $J$ Diabetes Res. 2018:ID 2786470. doi:10.1155/2018/2786470.

24. American Diabetes Association.Classification and diagnosis of diabetes. Sec. 2.In Standards of Medical Care in Diabetes-2015. Diabetes Care. 2015:38(Supplement. 1):S8-S16. 
25. Chobanian AV, Bakris GL, Black HR, et al. The seventh report of the joint national committee on prevention, detection, evaluation, and treatment of high blood pressure: the JNC 7 report [published correction appears in JAMA. 2003 Jul 9; 290(2):197]. JAMA. 2003;289(19):2560-2572. doi:10.1001/jama.289.19.2560

26. Trinder P. determination of glucose in blood using glucose oxidase with an alternative oxygen acceptor. J Ann Clin Biochem. 1969;6:2425. doi:10.1177/000456326900600108

27. Gonen B, Rubenstein A. Determination of glycohemoglobin. Diabetologia. 1978;15:1-5. doi:10.1007/BF01219319

28. Siedel J, Hagele E, Ziegenhorn J, et al. Reagent for the enzymatic determination of serum total cholesterol with improved lipolytic efficiency. Clin Chem. 1983;29:1075-1080. doi:10.1093/clinchem/29.6.1075

29. Friedwald WT, Levy RI, Fredrickson DS. Estimation of the concentration of low-denisty lipoprotein in plasma, without use of the preparative ultra-centrifuge. Clin Chem. 1972;18(6):499-502. doi:10.1093/clinchem/18.6.499

30. Levey AS, Coresh J, Greene T, et al. Using standardized serum creatinine values in the modification of diet in renal disease study equation for estimating glomerular filtration rate [published correction appears in ann intern med. 2008 Oct 7;149(7):519]. Ann Intern Med. 2006;145(4):247254. doi:10.7326/0003-4819-145-4-200608150-00004

31. Imig JD. Epoxyeicosatrienoic acids, hypertension, and kidney injury. Hypertension. 2015;65(3):476-482. doi:10.1161/ HYPERTENSIONAHA.114.03585

32. Capdevila JH, Pidkovka N, Mei S, et al. The Cyp2c44 epoxygenase regulates epithelial sodium channel activity and the blood pressureresponses to increased dietary salt. J Biol Chem. 2014;289(7):43774386. doi:10.1074/jbc.M113.508416
33. Gschwendtner A, Ripke S, Freilinger T, et al. Genetic variation in soluble epoxide hydrolase (EPHX2) is associated with an increased risk of ischemic stroke in white Europeans. Stroke. 2008;39(5):15931596. doi:10.1161/STROKEAHA.107.502179

34. Luo P, Zhou Y, Chang HH, et al. Glomerular 20-HETE, EETs, and TGF-beta1 in diabetic nephropathy. Am J Physiol Renal Physiol. 2009;296(3):F556-F563. doi:10.1152/ajprenal.90613.2008

35. Srivastava PK, Sharma VK, Kalonia DS, et al. Polymorphisms in human soluble epoxide hydrolase: effects on enzyme activity, enzyme stability, and quaternary structure. Arch BiochemBiophys. 2004;427 (2):164-169. doi:10.1016/j.abb.2004.05.003

36. Zhang L, Ding H, Yan J, et al. Genetic variation in cytochrome P450 $2 \mathrm{~J} 2$ and soluble epoxide hydrolase and risk of ischemic stroke in a Chinese population. Pharmacogenet Genomics. 2008;18(1):45-51. doi:10.1097/FPC.0b013e3282f313e8

37. Donnelly MK, Conley YP, Crago EA, et al. Genetic markers in the EET metabolic pathway are associated with outcomes in patients with aneurysmal subarachnoid hemorrhage. J Cereb Blood Flow Metab. 2015;35(2):267-276. doi:10.1038/jcbfm.2014.195

38. Katary MM, Pye C, Elmarakby AA. Meloxicam fails to augment the reno-protective effects of soluble epoxide hydrolase inhibition in streptozotocin-induced diabetic rats via increased 20-HETE levels. Prostaglandins Other Lipid Mediat. 2017;132:3-11. doi:10.1016/j. prostaglandins.2016.08.004

39. Askari A, Thomson SJ, Edin ML, et al. Roles of the epoxygenase CYP2J2 in the endothelium. Prostaglandins Other Lipid Mediat. 2013;107:56-63. doi:10.1016/j.prostaglandins.2013.02.003
The Application of Clinical Genetics

\section{Publish your work in this journal}

The Application of Clinical Genetics is an international, peerreviewed open access journal that welcomes laboratory and clinical findings in the field of human genetics. Specific topics include: Population genetics; Functional genetics; Natural history of genetic disease; Management of genetic disease; Mechanisms of genetic disease;

\section{Dovepress}

Counselling and ethical issues; Animal models; Pharmacogenetics; Prenatal diagnosis; Dysmorphology. The manuscript management system is completely online and includes a very quick and fair peerreview system, which is all easy to use. Visit http://www.dovepress. com/testimonials.php to read real quotes from published authors. 